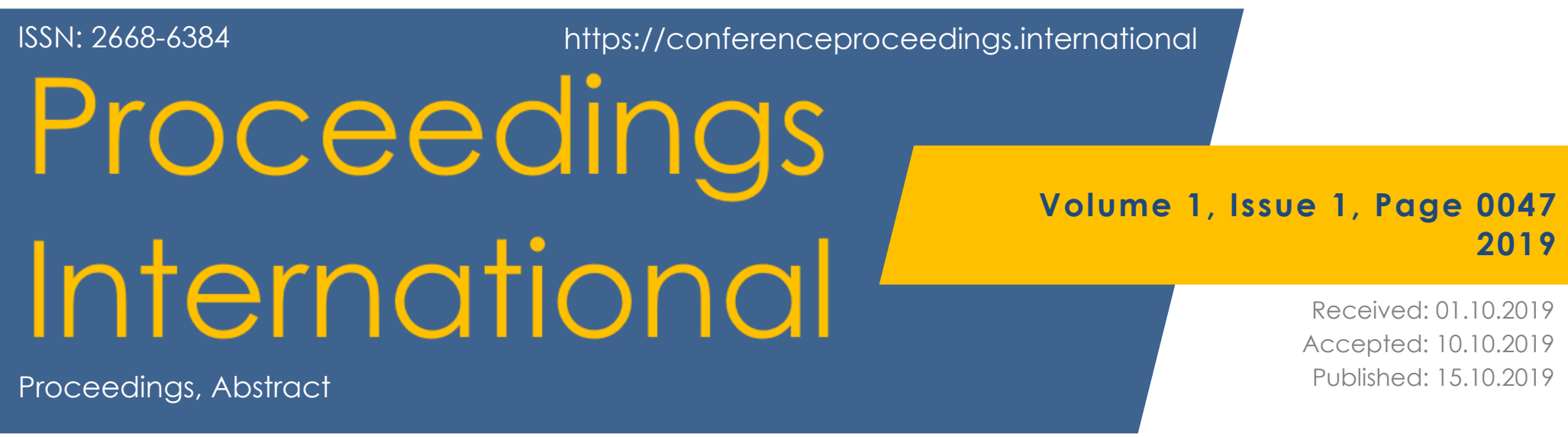

\title{
Enabling the multifunctionality of thin films through complex engineered nanoscale phases/nanodomains
}

\author{
N. D. Scarisoreanu 1, F. Andrei ${ }^{1}$, N. Enea ${ }^{1}$, I. Boerasu ${ }^{1}$, V. Ion ${ }^{1}$, R. Birjega ${ }^{1}$, M. Dinescu ${ }^{1}$, V. S. \\ Teodorescu ${ }^{2}$, C. Ghica ${ }^{2,}$, R.F. Negrea ${ }^{2}$ \\ 1 National Institute for Laser, Plasma and Radiation Physics, Magurele, Romania \\ 2 National Institute of Materials Physics, Magurele, Romania \\ * Correspondence: cghica@infim.ro; Scopus ID: 6603955468
}

$\mathrm{BiFeO}_{3}$ is one of the most promising multiferroic material exhibiting, however, drawbacks as low dielectric susceptibility, poor chemical stability and high dielectric loss. Recently, we have demonstrated that joining doping and epitaxial strain engineering a nanoscale stripe structure was induced, resulting in a significant improvement of the dielectric characteristics. By high resolution transmission electron microscopy (HR-TEM) we have evidenced nanostripe domains with alternating compressive and tensile strain in the $\mathrm{Y}$-doped $\mathrm{BiFeO}_{3}$ epitaxial thin films. Small band gap values of bismuth ferrite $(\mathrm{BFO})$ have triggered the interest due to the photovoltaic and photocatalytic potential, for pure or cation doped BFO. However, energy related properties of $\mathrm{BiFeO}_{3}$ can be improved by coupling the strong ferroelectricity of $\mathrm{BFO}$ with chemical stability and small band gap value of $\mathrm{LaFeO} 3$ within thickness graded heterostructures. Thin films of $\mathrm{BFO}$ on 5-20 nm thick epitaxial LFO films deposited on different strain-induced substrates, have been obtained by RHEED- assisted Pulsed Laser Deposition. Their functional dielectric, optical and photolytic behaviour has been unravelled in connexion with complex ensembles of nanoscale phase/nanodomain fluctuations within the epitaxial films.

Keywords: HR-TEM, thin films, nanoscale phases, $\mathrm{BiFe}_{3}$.

\section{Funding}

Not applicable.

\section{Acknowledgments}

Not applicable.

\section{Conflicts of Interest}

The authors declare no conflict of interest.

(C) 2019 by the authors. This article is an open access article distributed under the terms and conditions of the Creative Commons Attribution (CC BY) license (http://creativecommons.org/licenses/by/4.0/).

Cite This Work: Scarisoreanu, N.D.; Andrei, F.; Enea, N.; Boerasu, I.; Ion, V.; Birjega, R.; Dinescu, M.; Teodorescu, V.S.; Ghica, C.; Negrea, R.F. Enabling the multifunctionality of thin films through complex engineered nanoscale phases/nanodomains. Proceedings Int 2019, 1, 0047. 